\title{
ДИФФЕРЕНЦИАЛЬНАЯ ДИАГНОСТИКА И СРАВНИТЕЛЬНЫЕ РЕЗУЛЬТАТЫ КОМПЛЕКСНОГО ЛЕЧЕНИЯ ПАЦИЕНТОВ С ЭРОЗИВНЫМ И ЭРОЗИВНО-ЯЗВЕННЫМ ЭЗОФАГИТОМ
}

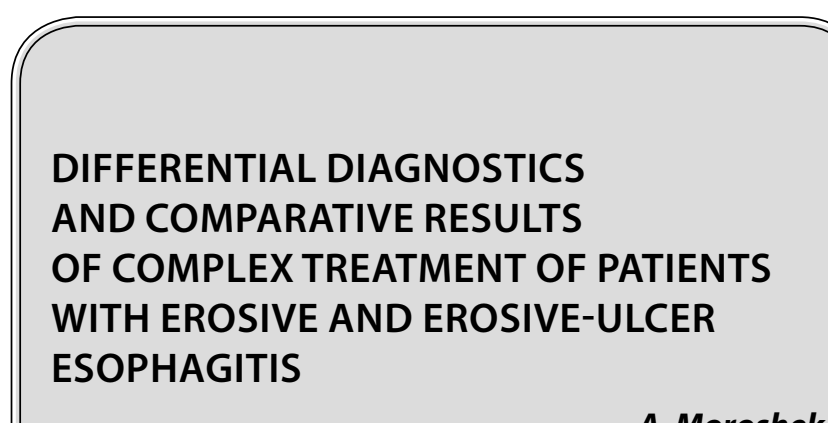

Summary. Objective. To assess the possibility of using a histological study for the differential diagnosis of erosive-ulcerative esophagitis and erosive esophagitis by assessing the operational characteristics of the method in identifying erosive-ulcerative esophagitis in patients with erosive esophagitis. To substantiate the feasibility of using the comprehensive treatment algorithm (CTA), which is based on the performance of antireflux surgery, in patients with erosive-ulcerative esophagitis by comparing the safety and effectiveness of using the CTA in patients with erosiveulcerative and erosive esophagitis. Materials and methods. The study was carried out on samples of 19 patients with erosive-ulcerative esophagitis (main group) and 282 patients with erosive esophagitis (control group). At the diagnostic stage, endoscopy, histological examination of mucosal samples and X-ray contrast study were used. At the stage of treatment - therapy with proton pump inhibitors and antireflux surgery. Results. Histological examination in patients with erosive esophagitis in order to identify erosive-ulcerative esophagitis, according to our data, has very high operational characteristics (sensitivity - 89.4\% (95\% Cl 76.9\% $-96.5 \%)$, specificity - 99.5\% (95\% Cl 98.1\% - 99.9\%)). There were no statistically significant differences between the study and control groups in the incidence of perioperative complications. Excellent and satisfactory long-term results of treatment were achieved in $94.7 \%$ (95\% (I 74\% - 99.9\%) of patients with erosive-ulcerative esophagitis. There were no statistically significant differences in the results of treatment between patients in the main and control groups. Conclusion. Histological examination can serve as a valuable method of clarifying and differential diagnosis of erosive-ulcerative esophagitis. Safety and efficacy (including in comparison with those in patients with erosive esophagitis) make it possible to recommend the CTA, including antireflux surgery, for the treatment of patients with erosive-ulcerative esophagitis.

Keywords: treatment of GERD complications; diagnosis of GERD complications; antireflux surgery; erosive reflux esophagitis; erosiveulcerative esophagitis.
Морошек Антон Александрович

К.м.Н., С.н.С., Приволжский филиал ФГБУ

«Национальный медицинский исследовательский чентр онкологии имени Н. Н. Блохина» Министерства здравоохранения Российской Федерации anton.moroshek@mail.ru

Аннотация. Цель исследования. Оценить возможность применения гистологического исследования для дифференциальной диагностики эрозивно-язвенного эзофагита и эрозивного эзофагита путем оценки операционных характеристик метода при выявлении эрозивно-язвенного эзофагита у пациентов с эрозивным эзофагитом. Обосновать целесообразность применения комплексного лечебного алгоритма, в основе которого лежит выполнение антирефлюксной операции, у пациентов с эрозивно-язвенным эзофагитом посредством сравнения безопасности и эффективности применения комплексного лечебного алгоритма у пациентов с эрозивно-язвенным и эрозивным эзофагитом. Материалы и методы. Исследование проведено на выборках из 19 пациентов с эрозивно-язвенным эзофагитом (основная группа) и 282 пациентов с эрозивным эзофагитом (контрольная группа). На диагностическом этапе применялась эндоскопия, гистологическое исследование образцов слизистой и рентгеноконтрастное исследование. На этапе лечения - терапия ингибиторами протоновой помпы и антирефлюксная операция. Результаты. Гистологическое исследование у пациентов с эрозивным эзофагитом с целью выявления эрозивно-язвенного эзофагита, по нашим данным, обладает очень высокими операционными характеристиками (чувствительность - 89,4\% (95\% дИ 76,9\%96,5\%), специфичность - 99,5\% (95\% ди 98,1\%-99,9\%)). Статистически значимые различия между основной и контрольной группами в частоте периоперационных осложнений отсутствовали. Отличные и удовлетворительные отдаленные результаты лечения были достигнуты у 94,7\% (95\% ДИ $74 \%$ - 99,9\%) пациентов с эрозивно-язвенным эзофагитом. Статистически значимые различия в результатах лечения между пациентами основной и контрольной групп отсутствовали. Заключение. Гистологическое исследование может служить ценным методом уточняющей и дифференциальной диагностики эрозивно-язвенного эзофагита. Безопасность и эффективность (в том числе и в сравнении с таковыми у пациентов с эрозивным эзофагитом), позволяют рекомендовать комплексный лечебный алгоритм, включающий антирефлюксную операцию, для лечения больных с эрозивно-язвенным эзофагитом.

Ключевые слова: лечение осложнений ГЭРБ; диагностика осложнений ГЭРБ; антирефлюксная хирургия; эрозивный рефлюкс-эзофагит; эрозивно-язвенный эзофагит. 


\section{Ввемение}

5 розивный рефлюкс-эзофагит (ЭЭ) осложняет хроническое течение гастроэзофагеальной рефлюксной болезни (ГЭРБ) в 7-12\% случаев и по разным оценкам может встречаться у 0,8-2\% населения развитых стран, нанося значительный социальный и экономический ущерб $[1,9,12]$. Хроническое течение нелеченного эрозивного эзофагита у 6-8\% пациентов приводит к поражению базальной мембраны с формированием эрозивно-язвенного эзофагита (ЭЯЭ), в свою очередь приводящему к таким грозным последствиям, как кровотечение (в 38\% случаев), формирование пептической стриктуры (в 12,5\% случаев) и перфорация пищевода (в 3,4\% случаев) [4].

В основе патогенеза ЭЭ и ЭЯЭ, как осложнений ГЭРБ лежит патологический гастроэзофагеальный рефлюкс (ГЭР), ведущий к продолжительному воздействию содержимого желудка на эпителий пищевода и вызывающий повреждающее действие, сходное с химическим ожогом $[10,11]$. При этом особую роль в нарушении клапанного механизма кардии и развитии (ГЭР) играет нарушение нормальных анатомо-физиологических взаимоотношений органов пищеводно-желудочного перехода, в особенности грыжа пищеводного отверстия диафрагмы (ГПОД) $[1,8]$.

Основой патогенетического подхода к ведению пациентов с ЭЭ, таким образом, видится лечение, направленное на восстановление клапанной функции и поддерживающих механизмов пищеводно-желудочного перехода [5, 6, 7]. Лапароскопические фундопликации (ЛФ), которые на сегодняшний день являются «золотым стандартом» антирефлюксной хирургии, применяются в отделении хирургии пищевода ГАУЗ «РКОД» МЗ РТ с 1997 года в составе комплексного лечебного алгоритма (КЛА) у пациентов с осложненными формами ГЭРБ [3]. В то время, как безопасность и эффективность КЛА при ЭЭ показана нами в более ранних исследованиях [2], целесообразность его применения при ЭяЭ требует подтверждения.

Развитие ЭяЭ является отправной точкой для формирования всех прочих тяжелых осложнений ЭЭ. Именно выявление и лечение пациентов с ЭяЭ играет основную роль в активной профилактике пищеводных кровотечений, пептической стриктуры и перфорации пищевода на фоне ГЭРБ. При этом дифференциальная диагностика между ЭЭ и ЭЯЭ может быть существенно затруднена при небольшой площади язв или выраженных отложениях фибрина [10].

Данное исследование посвящено изучению возможности применения гистологического исследования, как метода дифференциальной диагностики ЭЭ и ЭяЭ, а также оценке результатов комплексного лечения группы пациентов с ЭЯЭ, в том числе, и в сравнении с таковыми у пациентов с ЭЭ.

\section{Цель исслеАования}

Оценить возможность применения гистологического исследования для дифференциальной диагностики Эяэ и ЭЭ путем оценки операционных характеристик метода при выявлении ЭяЭ у пациентов с ЭЭ. Обосновать целесообразность применения комплексного лечебного алгоритма (КЛА), в основе которого лежит выполнение антирефлюксной операции, у пациентов с ЭЯЭ посредством сравнения безопасности и эффективности применения КЛА у пациентов с ЭЯЭ и с ЭЭ.

\section{Материалы и метолы}

Объектами изучения являлись пациенты с эрозивным эзофагитом, госпитализированные для обследования и лечения в отделение хирургии пищевода Государственного Автономного Учреждения здравоохранения «Республиканский клинический онкологический диспансер» Министерства здравоохранения Республики Татарстан (ГАУЗ «РКОД» МЗ РТ) с 01.01.1997 по 31.12.2013 г. (дата выбрана для обеспечения срока наблюдения не менее 72 месяцев у всех пациентов), которым в ходе лечения была выполнена антирефлюксная операция. Все участники исследования были жителями Российской Федерации. Исследование было одобрено Этическим комитетом ГАУЗ «РКОД» МЗ РТ.

По типу структуры и организации исследование аналитическое ретроспективное. Для исключения влияния других осложненных форм ГЭРБ при сравнительной оценке результатов лечения и безопасности лечебного алгоритма рассматривались только случаи ЭЭ и ЭЯЭ без сочетания с другими осложненными формами ГЭРБ.

Критерии включения в исследование: диагноз при поступлении - ЭЭ или ЭЯЭ, не сочетающийся с другими осложненными формами ГЭРБ; наличие показаний к выполнению хирургической антирефлюксной операции. Критерии исключения из исследования: наличие противопоказания к выполнению хирургической антирефлюксной операции; отказ от хирургической антирефлюксной операции по любым другим причинам; злокачественные новообразования в анамнезе. Конечные точки: окончание срока наблюдения после хирургической антирефлюксной операции, показания к повторной антирефлюксной операции.

Всего в исследование был включен 301 пациент, выбывших из исследования в течение срока наблюдения 
Таблица 1. Перечень и веса интраоперационных осложнений

\begin{tabular}{|l|l|}
\hline Интраоперационное осложнение & Вес интраоперационного осложнения \\
\hline Ранение внутрибрюшных органов & 1 \\
\hline Перфорация пищевода & 5 \\
\hline Кровотечение & 1 \\
\hline Пневмоторакс & 1 \\
\hline Повреждение вагуса & 5 \\
\hline Разрыв селезенки & 1 \\
\hline
\end{tabular}

Таблица 2. Перечень и веса ранних послеоперационных осложнений

\begin{tabular}{|l|l|}
\hline Раннее послеоперационное осложнение & Вес раннего послеоперационного осложнения \\
\hline Транзиторная дисфагия & 1 \\
\hline Gas-bloat-синдром & 1 \\
\hline Нагноение ран & 1 \\
\hline Плеврит & 1 \\
\hline Пневмония & 7 \\
\hline Кровотечение & 1 \\
\hline Свищ «манжетки» желудка & 7 \\
\hline Пневмоторакс & 1 \\
\hline Ишемические повреждения пищевода & 7 \\
\hline Тромбоэмболия ЛА & 7 \\
\hline Инфаркт миокарда & 7 \\
\hline Перитонит & 7 \\
\hline Медиастинит & 7 \\
\hline
\end{tabular}

не было. Пациентов с ЭЯЭ, составивших основную группу, было 19 (6,3\% (95\% ДИ 3,8\%-9,7\%)). Пациентов с ЭЭ без ЭЯЭ, составивших контрольную группу, - 282 (93,7\%, 95\% ДИ 90,3\% - 96,2\%). Срок наблюдения после выполнения антирефлюксной операции составил в основной группе от 72 до 274 месяцев, в контрольной группе от 72 до 278 месяцев.

При оценке операционных характеристик гистологического исследования при выявлении ЭяЭ у пациентов с ЭЭ дополнительно была рассмотрена группа из 117 пациентов с ЭЭ и ЭЯЭ в сочетании с другими осложненными формами ГЭРБ.

Диагноз ставился на основании: общеклинического обследования, эндоскопического исследования пищевода и желудка (ФЭГДС) с двойной хромоскопией растворами Люголя и метиленового синего и биопсией из участков подозрительных на метаплазию и краевых участков эрозий слизистой; полипозиционного рентгеноконтрастного исследования пищевода и пищеводно-желудочного перехода с контрастированием бариевой взвесью.

Основой разработанного и применяемого нами комплексного лечебного алгоритма (КЛА), использованного у пациентов основной и контрольной групп, были хирургические антирефлюксные вмешательства в различных вариантах. Мы выполняли антирефлюксные операции у всех пациентов с ЭЭ и ЭЯЭ, не имевших противопоказаний к операции.

Непосредственно КЛА состоял из 3 этапов: 1) консервативная антирефлюксная терапия длительностью 4-8 недель, включающая в себя регламентацию образа жизни, диету, медикаментозную антисекреторную терапию (ингибиторы протоновой помпы (ИПП) - омепразол или рабепразол по 20 мг 1-2 раза в день), эукинетики (эукинетики - домперидон по 20 мг внутрь 3 раза в сутки или итоприд по 50 мг внутрь 3 раза в сутки за 30 минут до приема пищи), с целью уменьшения выраженности воспалительных изменений слизистой пищевода и перивисцерита; 2) антирефлюксная хирургическая операция; 3) диспансерное наблюдение, включавшее ФЭГДС с двойной хромоскопией и биопсией и рентгеноконтрастное исследование пищевода и пищеводно-желудочного перехода в течение первых 12 месяцев срока наблюдения с частотой 1 раз в 6 месяцев, далее - с частотой 1 раз в год, либо по мере возникновения жалоб. При необходимости выполнялась остановка кровотечения путем в/в введения ИПП и эндоскопического гемостаза. 
Таблица 3. Перечень и веса поздних послеоперационных осложнений

\begin{tabular}{|l|l|}
\hline Позднее послеоперационное осложнение & Вес позднего послеоперационного осложнения \\
\hline Gas-bloat-синдром & 3 \\
\hline Стойкая дисфагия & 3 \\
\hline Диарея & 1 \\
\hline Свищ «манжетки» желудка & 3 \\
\hline Аэрофагия & 1 \\
\hline Феномен «телескопа» & 3 \\
\hline
\end{tabular}

Таблица 4. Операционные характеристики гистологического исследования при выявлении ЭЯЭ у пациентов с ГЭРБ

\begin{tabular}{|c|c|c|c|c|}
\hline & & \multicolumn{2}{|c|}{$\begin{array}{l}\text { Референсный метод } \\
\text { (эндоскопия) }\end{array}$} & \multirow[b]{2}{*}{ Всего } \\
\hline & & ЭЯЭ & Не ЭЯЭ & \\
\hline \multirow{3}{*}{ Исследуемый метод (гистологическое исследование) } & ЭЯЭ & 42 & 2 & 44 \\
\hline & Не ЭЯЭ & 5 & 369 & 374 \\
\hline & Итого & 47 & 371 & 418 \\
\hline \multicolumn{2}{|l|}{ Операционная характеристика } & Значение & \multicolumn{2}{|l|}{$95 \%$ ДИ } \\
\hline \multicolumn{2}{|l|}{ Чувствительность } & $89,36 \%$ & \multicolumn{2}{|c|}{$76,9 \%-96,45 \%$} \\
\hline \multicolumn{2}{|l|}{ Специфичность } & $99,46 \%$ & \multicolumn{2}{|c|}{$98,07 \%-99,93 \%$} \\
\hline \multicolumn{2}{|l|}{ Положительное отношение правдоподобия } & 165,77 & \multicolumn{2}{|c|}{$41,47-662,67$} \\
\hline \multicolumn{2}{|l|}{ Отрицательное отношение правдоподобия } & 0,11 & \multicolumn{2}{|c|}{$0,05-0,24$} \\
\hline
\end{tabular}

Все зарегистрированные в процессе лечения пациентов с ЭЭ и ЭяЭ осложнения были связаны с хирургическим этапом и его последствиями. Мы использовали информацию об интраоперационных осложнениях, ранних послеоперационных осложнениях (развившихся в срок до 3 месяцев после операции, срок в течение которого развившиеся осложнения ЛФ носили преимущественно транзиторный характер), поздних послеоперационных осложнениях (развившихся в срок от 3 месяцев до 1 года после операции) для разработки системы индексов, позволяющих обеспечить сопоставимость и сравнимость этих признаков у больных из разных групп. Осложнения были разделены на клинически значимые (однозначно ухудшают прогноз и утяжеляют течение заболевания и послеоперационного периода) и клинически малозначимые (имеют исключительно теоретическое и/или условное диагностическое значение). Каждому признаку был присвоен определенный коэффициент (вес). Веса клинически не значимых признаков принимали равными 1. Абсолютный индекс группы признаков для каждого отдельного пациента вычислялся, как сумма весов всех имеющихся у этого пациента признаков из данной группы, и характеризовал количество осложнений у данного пациента с учетом их тяжести. Относительный индекс (принимает значения от 0 до 1 включительно) вычислялся, как частное от деления абсолютного индекса на сумму всех весов из данной группы, то есть на максимально возможное значение абсолютного индекса, характеризовал выраженность осложнений у данного пациента относительно максимально возможной).

Перечень и веса наблюдавшихся интраоперационных осложнений приведены в таблице 1:

Перечень и веса наблюдавшихся ранних послеоперационных осложнений приведены в таблице 2:

Перечень и веса наблюдавшихся поздних послеоперационных осложнений приведены в таблице 3:

Результаты лечения разделяли на ближайшие (исход, отмеченный в течение первого года наблюдения) и отдаленные (исход, отмеченный в течение всего срока наблюдения после первого года). Для обеспечения сравнимости результатов лечения у пациентов различных групп к отличным результатам относили отсутствие в послеоперационном периоде потребности в дополнительных лечебных манипуляциях и приеме ИПП, удовлетворительными считали результаты лечения пациентов, которым в послеоперационном периоде потребовались эндоскопические лечебные манипуляции и/или длительный прием ИПП. При возникновении необходимости в повторном хирургическом вмешательстве результат лечения считался неудовлетворительным. 
В качестве порогового уровня статистической значимости было принято значение 0,05. Сравнение относительных частот признаков в 2 группах, а также в 3 и более группах с проводили применением критерия хи-квадрат Пирсона или приближенных формул. Границы доверительных интервалов (ДИ) для относительных частот (долей) вычислялись по методу Clopper-Pearson, точные значения уровней значимости вычисляли с помощью стандартных инструментов статистических программ. При анализе данных использовался пакет статистических программ STATISTICA 10.0 (StatSoft, США). Расчет характеристик диагностических процедур и их доверительных интервалов проводился в программе MedCalc 14.12.0 (MedCalc Software bvba).

\section{Результаты исслеАования}

Мы считали признаком наличия пептической язвы поражение слоев стенки пищевода глубже собственной пластинки слизистой. Кстати, следует отметить, что именно повреждение подслизистой основы признается спусковым механизмом в патогенезе такого тяжелого осложнения, как пептическая стриктура, что делает особо актуальной задачу своевременного обнаружения ЭяЭ. Стандартом диагностики (референсным методом) мы считали эндоскопическое исследование. После анализа результатов эндоскопии и гистологии у 418 пациентов с ЭЭ и ЭЯЭ были вычислены параметры, характеризующие диагностическую ценность гистологического исследования в выявлении ЭяЭ у пациентов с ЭЭ (Таблица 4).

Гистологическое исследование у пациентов с ЭЭ с целью выявления ЭЯЭ, по нашим данным, обладает очень высокими операционными характеристиками (чувствительность - 89,4\% (95\% ДИ 76,9\%-96,5\%), специфичность - 99,5\% (95\% ди 98,1\%-99,9\%). Гистологическое исследование в данном случае оказалось точным и ценным методом: нижняя граница 95\% ДИ положительного отношения правдоподобия, 41,47, указывает на то, что в сомнительных случаях гистологическое исследование может быть с успехом использовано для уточняющей и дифференциальной диагностики ЭЯЭ.

Медиана возраста пациентов с Эяэ на момент включения в исследование составила 52 года (95\% ДИ 46 лет - 72 года), с ЭЭ -50 лет 50 лет (95\% ДИ 48 лет 51 год). Мужчин в основной группе было (63,2\%, 95\% ДИ $38,4 \%-83,7 \%)$, в контрольной группе - (57,8\%, 95\% дИ 51,8\%-63,6\%). Грыжа пищеводного отверстия диафрагмы (ГПОД) имелась у 17 из 19 пациентов с ЭЯЭ (89,5\%, $95 \%$ ДИ 66,9\% - 98,7\%) и у 262 из 282 пациентов с ЭЭ (92,9\%, 95\% ДИ 89,3\% - 95,6\%), медиана длительности анамнеза ГЭРБ на момент поступления составляла в основной группе 20 месяцев (95\% ДИ 4-38 месяцев), в контрольной группе - 24 месяца (95\% ДИ 24-36 месяцев).
Статистически значимые различия в возрастно-половом составе, распространенности ГПОД и длительности анамнеза ГЭРБ между основной и контрольной группами отсутствовали.

Большинству пациентов (92,5\% пациентов с ЭЯэ и 95,7\% пациентов с ЭЭ) была выполнена антирефлюксная операция в виде лапароскопической фундопликации (ЛФ) по Ниссену-Розетти или Ниссену. Фундопликации в вариантах по Дору и Тупе, а также операции из лапаротомного доступа выполнялись при наличии показаний или технических сложностей (выраженный спаечный процесс, невозможность полной фундопликации, необходимость в конверсии в лапаротомию и т.д.). Статистически значимых различий в частоте применения различных видов хирургического доступа между пациентами с ЭяЭ и ЭЭ не отмечалось. Подавляющее большинство вмешательств (89,5\% (95\% ДИ 66,9\% $98,7 \%)$ у пациентов основной группы и 95,7\% (95\% ДИ $92,7 \%-97,8 \%)$ у пациентов контрольной группы) было выполнено с применением малоинвазивных подходов. В 2 случаях (10,5\%, 95\% ДИ 1,3\%-33,1\%) в группе ЭяЭ и в 11 случаях (3,9\%, 95\% ДИ 2\% - 6,9\%) в группе ЭЭ потребовалась конверсия в лапаротомию (в 7 случаях у пациентов с ЭЭ для коррекции интраоперационных осложнений, в 2 и 4 случаях у пациентов с ЭЯЭ и ЭЭ, соответственно,- вследствие выраженного спаечного процесса в брюшной полости). Статистически значимых различий в частоте конверсий в лапаротомию при выполнении лапароскопических антирефлюксных операций между пациентами основной и контрольной группы не отмечалось. У $1(0,4 \%)$ и пациента с ЭЭ операция была выполнена из лапаротомного доступа, причиной отказа от лапароскопии послужил выраженный спаечный процесс по данным анамнеза.

Средние значения относительных индексов интраоперационных (0,004 (95\% ДИ 0-0,008) для ЭяЭ и 0,11 (95\% ДИ 0,004-0,017) для ЭЭ, p>0,05), ранних послеоперационных $(0,008$ (95\% ДИ 0,002-0,014) для ЭяЭ и 0,012 (95\% дИ 0,009-0,014) для ЭЭ), p>0,05) и поздних послеоперационных $(0,011$ (95\% ДИ 0-0,022) для ЭЯЭ и 0,006 (95\% ДИ 0,002-0,01) для ЭЭ), р>0,05) осложнений имели очень низкие значения, что указывает на то, что большинство пациентов вообще не имели осложнений, а в случае наличия осложнений их выраженность была чрезвычайно далека от максимально возможной. И, в целом, комплексный лечебный алгоритм характеризовался высоким уровнем безопасности у пациентов с ЭЭ независимо от наличия ЭЯЭ. 95\% ДИ для всех индексов пересекаются, таким образом, при уровне значимости 0,05 статистически значимые различия в количестве и выраженности интраоперационных, а также ранних и поздних послеоперационных осложнений между пациентами с ЭЭ и ЭЯЭ отсутствовали. То есть комплексный 
Таблица 5. Сравнение ближайших результатов лечения пациентов с ЭЯЭ и ЭЭ.

\begin{tabular}{|c|c|c|c|c|c|}
\hline \multirow[b]{2}{*}{$\begin{array}{l}\text { Ближайший результат } \\
\text { лечения }\end{array}$} & \multicolumn{2}{|l|}{ ЭЯЭ } & \multicolumn{2}{|l|}{$\ni \ni$} & \multirow[b]{2}{*}{ Значение p } \\
\hline & $\begin{array}{l}\text { Относитель- } \\
\text { ная частота } \\
\text { результата,\% }\end{array}$ & 95\% ди & $\begin{array}{l}\text { Относитель- } \\
\text { ная частота } \\
\text { результата,\% }\end{array}$ & 95\% ди & \\
\hline Отличный & $89,5 \%$ & $66,9 \%-98,7 \%$ & $94,3 \%$ & $90,9 \%-96,7 \%$ & 0,3939 \\
\hline Удовлетворительный & $0,0 \%$ & $0 \%-17,6 \%$ & $0,7 \%$ & $0,1 \%-2,5 \%$ & 0,7144 \\
\hline Неудовлетворительный & $10,5 \%$ & $1,3 \%-33,1 \%$ & $5,0 \%$ & $2,7 \%-8,2 \%$ & 0,3023 \\
\hline
\end{tabular}

Таблица 6. Сравнение отдаленных результатов лечения пациентов с ЭЯЭ и ЭЭ.

\begin{tabular}{|c|c|c|c|c|c|}
\hline \multirow[b]{2}{*}{$\begin{array}{l}\text { Ближайший результат } \\
\text { лечения }\end{array}$} & \multicolumn{2}{|l|}{ ЭЯว } & \multicolumn{2}{|l|}{$\ni \ni$} & \multirow[b]{2}{*}{ Значение p } \\
\hline & $\begin{array}{l}\text { Относитель- } \\
\text { ная частота } \\
\text { результата,\% }\end{array}$ & 95\% ди & $\begin{array}{l}\text { Относитель- } \\
\text { ная частота } \\
\text { результата,\% }\end{array}$ & 95\% Ди & \\
\hline Отличный & $94,7 \%$ & $74 \%-99,9 \%$ & $94,7 \%$ & $91,4 \%-97 \%$ & 1 \\
\hline Удовлетворительный & $0,0 \%$ & $0 \%-17,6 \%$ & $0,0 \%$ & $0 \%-1,3 \%$ & 1 \\
\hline Неудовлетворительный & $5,3 \%$ & $0,1 \%-26 \%$ & $5,3 \%$ & $3 \%-8,6 \%$ & 1 \\
\hline
\end{tabular}

лечебный алгоритм был одинаково безопасен у пациентов с ЭЭ независимо от наличия ЭЯЭ.

Сравнение ближайших результатов применения КЛА при лечении пациентов с ЭЯЭ и ЭЭ представлено в таблице 5.

При уровне значимости 0,05 статистически значимой разницы в ближайших результатах лечения между пациентами с ЭЭ и ЭЯЭ не отмечалось.

Сравнение отдаленных результатов применения КЛА при лечении пациентов с ЭЯЭ и ЭЭ представлено в таблице 6.

При уровне значимости 0,05 статистически значимой разницы в отдаленных результатах лечения между пациентами с ЭЯЭ и ЭяЭ также отмечено не было.

\section{Зак^ючение}

Нами на большом клиническом материале показано, что ЭяЭ характеризуется специфическими изменениями (такими, как повреждение слоев стенки глубже собственной пластинки слизистой), выявляемыми при гистологическом исследовании. А само гистологическое исследование обладает высокими операционными характеристиками и может быть ценным методом уточняющей и дифференциальной диагностики ЭЯЭ и ЭЭ. С практической точки зрения это означает, что ЭЯЭ на фоне ЭЭ может быть выявлен при минимальной площади поражения слизистой, а значит, появляется возможность скорректировать лечебную тактику, чтобы максимально снизить риск развития тяжелых осложнений, таких как пептическая стриктура, кровотечение и перфорация пищевода.

Наши данные указывают на то, что ГПОД, ЭЭ и ЭЯэ можно рассматривать в качестве сопутствующих друг другу заболеваний: у 92,9\% пациентов с ЭЭ и 89,5\% пациентов с ЭяЭ имелась ГПОД. Эффективная коррекция ГПОД возможна только оперативным путем, таким образом, крайне высокая распространенность ГПОД у пациентов с ЭЭ и ЭяЭ указывает на целесообразность обязательного включения антирефлюксной операции в алгоритм лечения данной патологии.

Крайне низкие значения относительных индексов интраоперационных, ранних и поздних послеоперационных осложнений (верхняя граница 95\% ДИ менее 0,023) указывают на то, что выраженность периоперационных осложнений у пациентов с ЭяЭ составляет не более $2,3 \%$ от максимально возможной, а применение КЛА можно охарактеризовать, как безопасное при лечении пациентов с ЭЯЭ. Отсутствие статистически значимой разницы в значениях относительных индексов периоперационных осложнений у пациентов с ЭЯЭ и ЭЭ указывает на то, что наличие ЭяЭ не приводит к повышению частоты осложнений при использовании КЛА в лечении пациентов с ЭЭ.

КЛА показал высокую эффективность в лечении пациентов с ЭЯЭ: отличных и удовлетворительных результатов удалось достичь в 94,7\% случаев (95\% ДИ 
74\% - 99,9\%). При этом присутствие ЭЯэ у пациентов с ЭЭ не приводило к ухудшению результатов лечения.

Таким образом, безопасность и эффективность КЛА (в том числе и в сравнении с таковыми у паци- ентов с ЭЭ), а также целесообразность выполнения хирургических антирефлюксных операций у всех пациентов с ЭЯЭ позволяют рекомендовать КЛА, включающий антирефлюксную операцию, для лечении больных с ЭЯЭ.

\section{ЛИТЕРАТУРА}

1. Бордин, Д. С. Гастроэзофагеальная рефлюксная болезнь: от патогенеза к лечению / Д. С. Бордин, С. В. Колбасников // Лечащий Врач. — 2018. — № 7. —C. 34.

2. Морошек, А. А. Комплексный подход в лечении пациентов с осложнёнными формами гастроэзофагеальной рефлюксной болезни / А. А. Морошек, М. В. Бурмистров // Казанский медицинский журнал.— 2020.— Т. 101.— № 4. — С. 595-602.

3. Профилактика осложнений при лапароскопических антирефлюксных операциях / Т. Л. Шарапов, Е. И. Сигал, А. А. Морошек [и др.] // Эндоскопическая хирургия.-2012.— № 3.- С. 9-14.

4. Распространенность эрозивного эзофагита и пептических стриктур пищевода по данным эндоскопических исследований / 0. Ж. Пензина, С. С. Потхоева, В. В. Суворова [и др.] // Медицина завтрашнего дня: материалы XVII межрегион. науч.-практ. конф. студентов и молодых ученых: сб. науч. тр.— Чита, 2018. - C. 93-94.

5. Тарбаев, И. С. Нерешенные вопросы хирургического лечения гастроэзофагеальной рефлюксной болезни / И. С. Тарбаев, Д. И. Василевский, А. М. Ахматов // Вестник хирургии имени И. И. Грекова. — 2018. - Т. 177, № 4. — С. 98-100.

6. Юрасов, А. В. Показания к оперативному лечению рефлюкс-эзофагита / А. В. Юрасов, А. Л. Шестаков, Т. Т. Битаров // Доказательная гастроэнтерология.2018. - T. 7, № 2. - C. 30-34.

7. Bansal, A. Treatment of GERD complications (Barrett's, peptic stricture) and extra-oesophageal syndromes / A. Bansal, P. J. Kahrilas // Best practice \& research. Clinical gastroenterology. - 2010.—Vol. 24.—P. 961-968.

8. Evidence-based practice guideline for surgical treatment of gastroesophageal reflux disease 2018 / H. S. Seo, M. Choi, S.-Y. Son [et al.] // Journal of gastric cancer.2018. - Vol. 18, № 4. - P. 313-327.

9. Guidelines for the Diagnosis and Management of Gastroesophageal Reflux Disease / M. E. Karim, S. Rahim, M. Mahmuduzzaman [et al.] // Journal of Shaheed Suhrawardy Medical College.—2017.—Vol. 8, № 1.—P. 30-33.

10. Review article: oesophageal complications and consequences of persistent gastro-oesophageal reflux disease / J. Pisegna, G. Holtmann, C. W. Howden [et al.] // Alimentary pharmacology \& therapeutics. - 2004. - Vol. 20, Suppl 9. - P. 47-56.

11. Role of the lower esophageal sphincter and hiatal hernia in the pathogenesis of gastroesophageal reflux disease / M. Fein, M. P. Ritter, T. R. DeMeester [et al.] // Journal of gastrointestinal surgery: official journal of the Society for Surgery of the Alimentary Tract. — 2005. — Vol. 3. — P. 405-410.

12. Sonnenberg, A. Clinical epidemiology and natural history of gastroesophageal reflux disease / A. Sonnenberg, H. B. El-Serag // The Yale journal of biology and medicine. - 2005.—Vol.72.- P. 81-92.

(c) Морошек Антон Александрович ( anton.moroshek@mail.ru ).

Журнал «Современная наука: актуальные проблемы теории и практики» 